\title{
Bicubic Polar Subdivision
}

\author{
K. Karčiauskas \\ Vilnius University \\ and \\ J. Peters \\ University of Florida
}

\begin{abstract}
We describe and analyze a subdivision scheme that generalizes bicubic spline subdivision to control nets with polar structure. Such control nets appear naturally for surfaces with the combinatorial structure of objects of revolution and at points of high valence in subdivision meshes. The resulting surfaces are $C^{2}$ except at a finite number of isolated points where the surface is $C^{1}$ and the curvature is bounded.

Categories and Subject Descriptors: I.3.5 []: Computational Geometry and Object Modeling; J.6 []: Computer-Aided Engineering

General Terms: Algorithms

Additional Key Words and Phrases: Subdivision, polar layout, polar net, bicubic, Catmull-Clark, curvature continuity
\end{abstract}

\section{INTRODUCTION}

Polar control nets (Figure 1) capture the combinatorial structure of objects of revolution and are therefore more natural at points of high valence (see e.g. Figure 2) than the all-quads layout favored by Catmull-Clark subdivision [Catmull and Clark 1978]. Correspondingly, we define and analyze in the following a binary subdivision scheme that, just like Catmull-Clark subdivision, generalizes the refinement rules of uniform cubic splines - but for the layout of a polar net.

Formally, a control net without boundary is a polar net [Karčiauskas and Peters 2007] if it consists of extraordinary mesh nodes surrounded by triangles, and of quadrilaterals that have nodes of valence four. The extraordinary mesh nodes need only be separated by one layer of nodes of valence four as illustrated in Figure 7, left. Applying quad-tri subdivision [Stam and Loop 2003; Peters and Shiue 2004; Schaefer and Warren 2005] to a polar net is not a good alternative, since Loop subdivision also

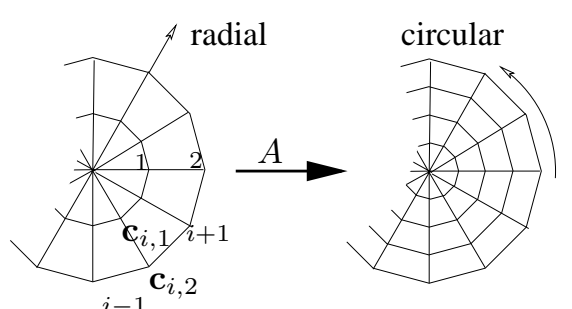

Fig. 1. Polar control net near an extraordinary point (left) and its refinement (right) under subdivision. The control points $\mathbf{c}_{i j}$ have subscripts $i$ indicating (modulo the valence $n$ ) the direction and subscripts $j$ indicating the radial distance to the extraordinary point $\mathbf{c}_{i 0}$. Only the radial, not the circular direction is refined.

does not cope well with such input meshes (Figure 2). Polar subdivision differs structurally from tensored univariate schemes with singularities, e.g. [Morin et al. 2001], in that the number of neighbors of the extraordinary point does

Permission to make digital/hard copy of all or part of this material without fee for personal or classroom use provided that the copies are not made or distributed for profit or commercial advantage, the ACM copyright/server notice, the title of the publication, and its date appear, and notice is given that copying is by permission of the ACM, Inc. To copy otherwise, to republish, to post on servers, or to redistribute to lists requires prior specific permission and/or a fee.

(c) 20YY ACM 0730-0301/20YY/0100-0001 \$5.00 

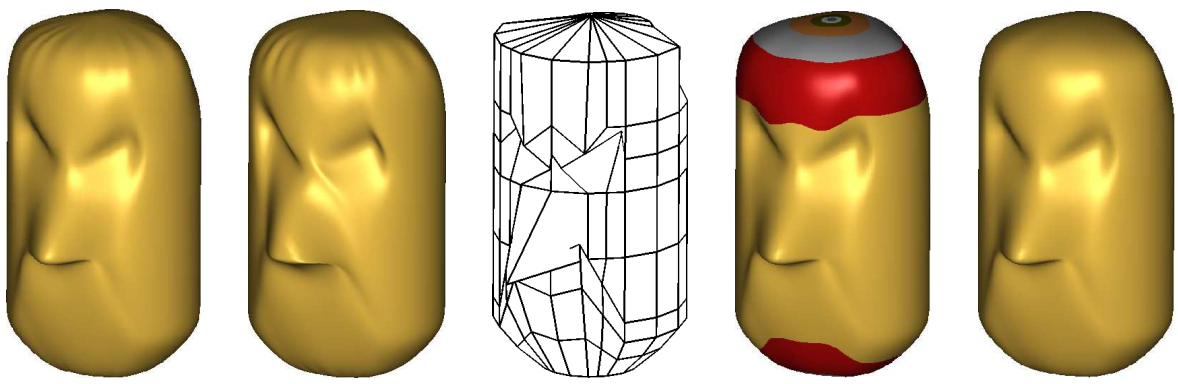

Fig. 2. Wrinkle removal on an Easter Island head (valence 20). (from left to right) Catmull-Clark subdivision, Loop subdivision (quad facets are split), control net, color-coded rings of the polar subdivision surface, polar subdivision surface.

not double with each polar subdivision step but stays fixed. Quadrilaterals in a polar net are not split and the control net refines only towards the extraordinary point (Figure 1). Therefore, the polar control net does not, off hand, serve the function of approximating the surface ever more closely by smaller facets. However, the resulting surface as a single B-spline mesh growing towards the extraordinary point, i.e. the surface does not have the cascading sequence of T-corners intrinsic to Catmull-Clark surfaces. Section 4, Control Nets, explains this in detail.

Compared to [Karčiauskas et al. 2006], the more localized computation of bicubic polar subdivision results in a more localized curvature distribution. At the extraordinary point, the curvature of surfaces generated by bicubic polar subdivision is only bounded but need not be continuous while the algorithm in [Karčiauskas et al. 2006] generates $C^{2}$ surfaces. The present scheme has, however, the advantage of simpler rules without visibly sacrificing good shape.

\section{POLAR REFINEMENT RULES}

Apart from the extraordinary mesh nodes, the polar net defined in the introduction, is a standard bicubic B-spline control net. For the layer of quadrilaterals adjacent to the triangles, we interpret the triangles as degenerate quadrilaterals with one edge collapsed. It is easy to check, for example by conversion to Bézier form, that this interpretation does not result in singularities in the quadrilateral layer. In order to map a polar net to a refined polar net, we will refine the bicubic spline net only in the radial direction (cf. Figure 1).
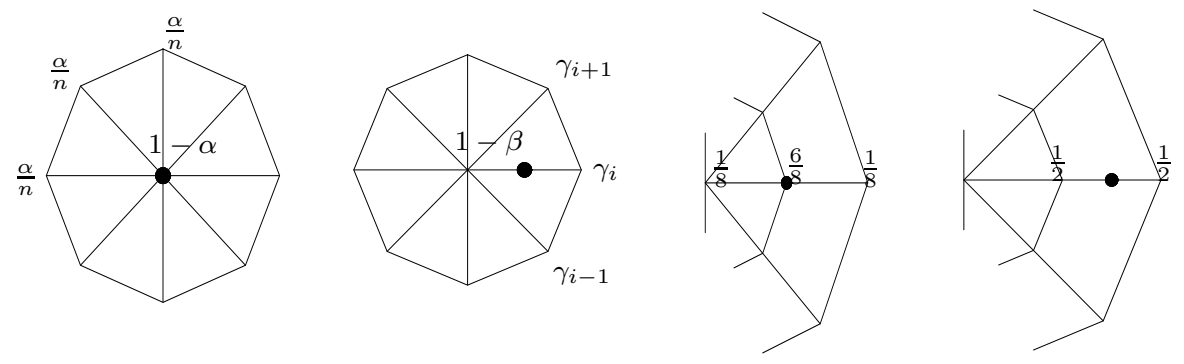

Fig. 3. Refinement stencils for binary polar subdivision.

As is typical for subdivision algorithms, we need only explain how to refine the polar net immediately connected to extraordinary mesh nodes. To obtain leading eigenvalues $1, \frac{1}{2}, \frac{1}{2}, \frac{1}{4}, \frac{1}{4}, \frac{1}{4}$, it suffices to have special rules only at the extraordinary mesh node and its direct neighbors (Figure 3). The two regular rules are the subdivision rules for 
univariate uniform cubic splines. The extraordinary rules have the weights

$$
\alpha:=\beta-\frac{1}{4}, \quad \beta:=\frac{1}{2}, \quad \gamma_{k}:=\frac{1}{n}\left(\beta-\frac{1}{2}+\frac{5}{8} c_{n}^{k}+\left(c_{n}^{k}\right)^{2}+\frac{1}{2}\left(c_{n}^{k}\right)^{3}\right), \quad c_{n}^{k}:=\cos \left(\frac{2 \pi k}{n}\right) .
$$

Here, we chose $\beta=1 / 2$ to emphasize convexity at the extraordinary point, since this is likely the dominant scenario for polar meshes. This choice is also reasonable for saddles (Figure 9). Section 4, Convexity and Valence discusses the role of $\beta$ in more detail.

A useful property of polar surfaces is that the valence can be changed by interpreting each circular ring of coefficients as the control polygon of a cubic spline curve. To avoid a special discussion of low valences, we uniformly insert knots in the circular spline curves and double the valence when $n \in\{3,4,5\}$. That is, we may assume $n \geq 6$ in the following.

\section{PROPERTIES BY CONSTRUCTION}

Let $\mathbf{c}_{i, j}^{m}$ be the control point of the $i$ th sector and the $j$ th layer as indicated in Figure 1 . The central node is considered split into $n$ copies $\mathbf{c}_{i 0}^{m}$, each weighted by $1 / n$. Then the vector of control points

$$
\mathbf{c}^{m}:=\left(\ldots, \mathbf{c}_{i 0}^{m}, \mathbf{c}_{i 1}^{m}, \mathbf{c}_{i 2}^{m}, \mathbf{c}_{i 3}^{m}, \ldots\right) \in \mathbb{R}^{4 n \times 4 n},
$$

is refined by a subdivision matrix with block-circulant structure: $\mathbf{c}^{m+1}=A \mathbf{c}^{m}$,

$$
A:=\left(\begin{array}{cccc}
A_{0} & A_{1} & \ldots & A_{n-1} \\
A_{n-1} & A_{0} & \ldots & A_{n-2} \\
\vdots & & \ddots & \vdots \\
\dot{A}_{1} & \ldots & A_{n-1} & A_{0}
\end{array}\right) \in \mathbb{R}^{4 n \times 4 n}, A_{0}:=\left(\begin{array}{ccccc}
\frac{1-\alpha}{n} & \frac{\alpha}{n} & 0 & 0 \\
\frac{1-\beta}{n} & \gamma_{0} & 0 & 0 \\
\frac{1}{8 n} & \frac{3}{4} & \frac{1}{8} & 0 \\
0 & \frac{1}{2} & \frac{1}{2} & 0
\end{array}\right), A_{i}:=\left(\begin{array}{cccc}
\frac{1-\alpha}{n} & \frac{\alpha}{n} & 0 & 0 \\
\frac{1-\beta}{n} & \gamma_{i} & 0 & 0 \\
\frac{1}{8 n} & 0 & 0 & 0 \\
0 & 0 & 0 & 0
\end{array}\right), i=1, \ldots, n-1,
$$

that can be block-diagonalized by Discrete Fourier Transform $\hat{A}_{i}:=\sum_{k=0}^{n-1} \omega_{n}^{i k} A_{k}, \quad \omega_{n}^{\ell}:=\exp \left(\frac{2 \pi \ell \sqrt{-1}}{n}\right)$, so that the eigen-analysis is pleasantly simple.

LEMMA 1. For generic input data, the limit surface of bicubic polar subdivision is $C^{2}$ except at isolated extraordinary points where the surface is $C^{1}$ and the curvature bounded.

PROOF. As illustrated in Figure 4, control point layers 1 through 5 define two rings of bicubic splines (Figure 4 middle). This double-ring is $C^{2}$ since it corresponds to a regular (periodic) tensor-product spline. As in Catmull-Clark subdivision, consecutive double-rings join $C^{2}$. For $n>5$,

$$
\hat{\gamma}_{i}=\left\{\begin{array}{ll}
\frac{1}{2}, & \text { if } i \in\{1, n-1\} \\
\frac{1}{4}, & \text { if } i \in\{2, n-2\} \\
\frac{1}{16}, & \text { if } i \in\{3, n-3\} \\
0, & \text { if } i>3 \text { and } i<n-3 .
\end{array} \quad \text { and } \hat{A}_{0}=\left(\begin{array}{cccc}
1-\alpha & \alpha & 0 & 0 \\
1-\beta & \beta & 0 & 0 \\
\frac{1}{8} & \frac{3}{4} & \frac{1}{8} & 0 \\
0 & \frac{1}{2} & \frac{1}{2} & 0
\end{array}\right), \hat{A}_{i}=\left(\begin{array}{cccc}
0 & 0 & 0 & 0 \\
0 & \hat{\gamma}_{i} & 0 & 0 \\
0 & \frac{3}{4} & \frac{1}{8} & 0 \\
0 & \frac{1}{2} & \frac{1}{2} & 0
\end{array}\right) .\right.
$$

The eigenvalues of $\hat{A}_{0}$ are $1, \frac{1}{4}, \frac{1}{8}, 0$ and the eigenvalues of $\hat{A}_{i}$ for $i=1, \ldots, n-1$, are $\hat{\gamma}_{i}:=\sum_{k=0}^{n-1} \omega_{n}^{i k} \gamma_{k}, \frac{1}{8}, 0,0$. In particular, $\lambda_{1}=\hat{\gamma}_{1}$ and $\left(\lambda_{1}\right)^{2}=\lambda_{2}=\hat{\gamma}_{2}$ as is required for bounded curvature.

Since the eigenvector of matrix $\hat{A}_{1}$ for $\lambda_{1}=\frac{1}{2}$ is $(0,1,2,3)^{\mathrm{t}}$, the subdominant eigenvectors of $A$ are the coordinates of

$$
\mathbf{v}=\left(\ldots, \mathbf{r}_{3}^{i-1}, \mathbf{r}_{0}^{i}, \mathbf{r}_{1}^{i}, \mathbf{r}_{2}^{i}, \mathbf{r}_{3}^{i}, \mathbf{r}_{0}^{i+1}, \ldots\right), \quad \mathbf{r}_{k}^{i}:=k\left[\begin{array}{c}
\cos i \frac{2 \pi}{n} \\
\sin i \frac{2 \pi}{n}
\end{array}\right], \quad i=1, \ldots, n, k=0,1,2,3 .
$$

The control net $\mathbf{v}$ defines the characteristic map (Figure 4, middle) [Reif 1995], whose regularity and injectivity are easily verified [Peters and Reif 1998; Umlauf 1999]. The eigenvectors corresponding to the eigenvalue 1/4 are from Fourier blocks 0, 2 and $n-2$ and they are not generalized eigenvectors. Explicitly, for use in Section 4, the 

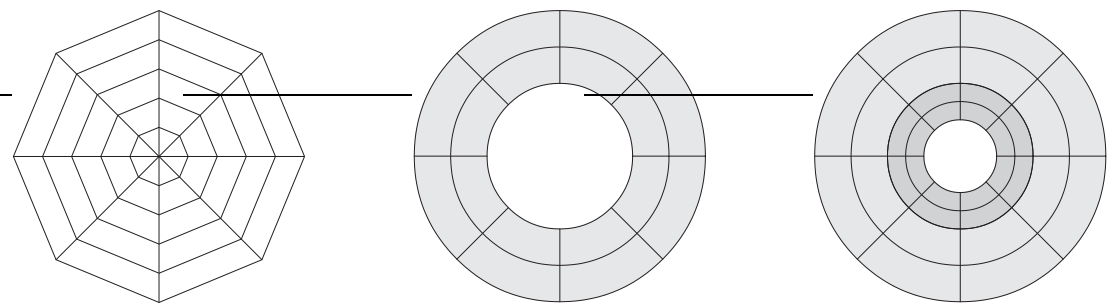

Fig. 4. (left) Layers 0 through 5 (generated by one subdivision of layers 0 through 3) define (middle) one piecewise bicubic double-ring. (right) Consecutive double-rings join smoothly and, unlike Catmull-Clark subdivision, without T-corners.

eigenvectors $v_{2 k}$ to the eigenvalue $\frac{1}{4}$ of $\hat{A}_{k}$ for Fourier index $k \in\{0,2\}$ are

$$
v_{20}:=(1+3 b, 1,7+3 b, 16+6 b)^{\mathrm{t}}, \quad b:=\frac{1}{4 \beta-1}, \quad v_{22}:=(1,4,6,14)^{\mathrm{t}} .
$$

Together with the curvature bounded spectrum, this implies curvature boundedness as claimed.

LEMMA 2. The limit extraordinary point is

$$
\eta \mathbf{c}_{00}+(1-\eta) \frac{1}{n} \sum_{i=1}^{n} \mathbf{c}_{i 1} \quad \eta:=\frac{4(1-\beta)}{3} .
$$

Proof. We choose the representation $\bar{A} \in \mathbb{R}^{3 n+1 \times 3 n+1}$ of the subdivision operator where we do not replicate the central node $c_{00}$ :

$$
\bar{A}:=\left(\begin{array}{cccc}
1-\alpha & \mathbf{a}_{r} & \ldots & \mathbf{a}_{r} \\
\mathbf{a}_{c} & \bar{A}_{0} & \ldots & \bar{A}_{n-1} \\
\vdots & & \ddots & \vdots \\
\mathbf{a}_{c} & \ldots & \bar{A}_{n-1} & \overline{\bar{A}}_{0}
\end{array}\right) \quad \mathbf{a}_{r}:=\left[\frac{\alpha}{n}, 0,0\right] \quad \mathbf{a}_{c}:=\left[1-\beta, \frac{1}{8}, 0\right]^{\mathrm{t}} \quad \bar{A}_{0}:=\left(\begin{array}{ccc}
\gamma_{0} & 0 & 0 \\
\frac{3}{4} & \frac{1}{8} & 0 \\
\frac{1}{2} & \frac{1}{2} & 0
\end{array}\right) \quad \bar{A}_{i}:=\left(\begin{array}{ccc}
\gamma_{i} & 0 & 0 \\
0 & 0 & 0 \\
0 & 0 & 0
\end{array}\right), i=1, \ldots, n-1 .
$$

We can directly check that the left eigenvector of $\bar{A}$ with respect to the dominant eigenvalue 1 is

$$
\left[\frac{1-\beta}{1-\beta+\alpha}, \boldsymbol{\ell}, \boldsymbol{\ell}, \ldots, \boldsymbol{\ell}\right]^{\mathrm{t}}, \quad \boldsymbol{\ell}:=\left[\frac{\alpha}{n(1-\beta+\alpha)}, 0,0\right] .
$$

The claim follows (see [DeRose et al. 1998], Appendix A) since the entries sum to 1.

Since $[0,1,0,0]$ is a left eigenvector to $\hat{A}_{1}$, the normal direction at the extraordinary point is simply $\left(\sum_{i=1}^{n} \cos i \frac{2 \pi}{n} \mathbf{c}_{i 0}\right) \times\left(\sum_{i=1}^{n} \sin i \frac{2 \pi}{n} \mathbf{c}_{i 0}\right)$.

\section{DISCUSSION}

This section discusses some alternative schemes, the meaning of control polyhedra and adjustment of valence and convexity.

\section{Alternative Schemes.}

The bicubic polar subdivision algorithm has special rules for both the new central node and its direct neighbors. Choosing symmetric special rules only for the central node does not yield appropriate degrees of freedom for smoothness. Specifically, forcing a double subdominant eigenvalue by tuning only the rules for the central node, leads to one single subdominant eigenvector for $n>3$; only for $n=3$, do there exist rules to generate $C^{1}$ surfaces with a spectrum suitable for bounded curvature. So, a direct polar analogue of Catmull-Clark subdivision fails and the question arises whether a ternary polar subdivision scheme, analogous to [Loop 2002], is advantageous. 

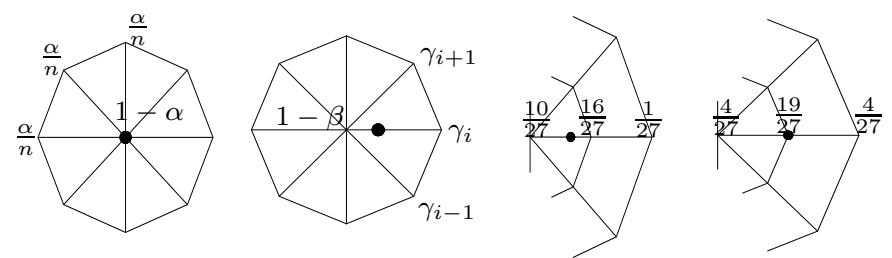

Fig. 5. Refinement stencils for a ternary polar subdivision (splitting into three in the radial direction) where $\beta:=\frac{38}{81}, \alpha:=\beta-\frac{1}{9}, \gamma_{k}:=$ $\frac{4}{81 n}\left(5+2 c_{n}^{k}\right)\left(1+c_{n}^{k}\right)^{2}$.

We derived such a variant for comparison (see Figure 5). The weights $\gamma_{k}$ are non-negative and the scheme satisfies all the constraints on the leading eigenvalues $\left(1, \frac{1}{3}, \frac{1}{3}, \frac{1}{9}, \frac{1}{9}, \frac{1}{9}\right)$ and eigenvectors for curvature boundedness. However, the resulting surfaces did not look better than those of the proposed binary subdivision.

Control Nets and Surface Rings.

Subdivision surfaces can either be viewed as refining a control net or as generating a sequence of surface rings converging to the extraordinary point [Reif 1995]. The first serves intuition if the control net outlines the shape, the second is preferred for exact evaluation, computing and analysis. Both Catmull-Clark subdivision and polar subdivision admit the two views but differ in their bias. To see this, define a T-corner to be the location where an edge between two distinct polynomial patches meets the midpoint of an edge of a third. With each refinement, Catmull-Clark subdivision generates T-corners between the patches of adjacent surface rings (Figure 6, left top). Polar subdivision does not generate T-corners (Figure 6, right top) since the control net refines only towards the extraordinary point (Figure 1). One approach for generating a faceted approximation converging to the underlying surface is to split the quadrilaterals of the polar net at each refinement into four and leave the triangles untouched. This yields T-corners in the faceted approximation (Figure 6, right bottom). Reflecting the bias towards presenting a mesh without $\mathrm{T}$ corners versus obtaining a surface without T-corners, Catmull-Clark subdivision is usually illustrated by a sequence of control nets (Figure 1,left bottom), hiding the surface T-corners, while polar surfaces are preferably introduced as a sequence of surface rings.

Convexity and Valence.

Decreasing the parameter $\beta$ in (1) pulls the surface closer to the extraor-

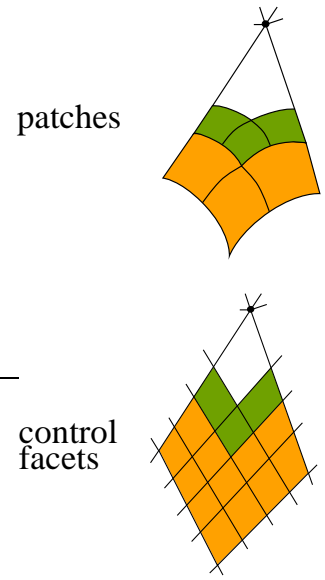

Catmull-Clark
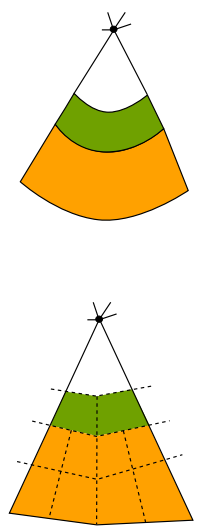

polar

Fig. 6. Layout of patches and control polyhedron for Catmull-Clark subdivision (left) and polar subdivision (right). The Tcorners in Catmull-Clark (left top) are intrinsic (the coarser patch is $C^{\infty}$ at the Tcorner). The T-corners in the refined polyhderon (right bottom) are optional and not part of the polar net.

dinary mesh node. Recently [Ginkel and Umlauf 2006] documented how such straightforward manipulation results in a limit surface in the desirable region of a 'shape chart' [Karciauskas et al. 2004]: decreasing $\beta$ emphasizes convexity. We therefore chose $\beta:=$ $1 / 2$ (see Figure 10) over $\beta:=5 / 8$ even though the latter yields non-negative weights $\gamma_{k}=\frac{1}{8 n}\left(1+c_{n}^{k}\right)\left(1+2 c_{n}^{k}\right)^{2} \geq 0$. Table I shows the effect of $\beta$ on the subsubdominant eigenvector $v_{20}$ of (4), that determines the shape in the convex setting, and its second difference $\Delta v_{20}$. For $\beta=1 / 2$, the sector partition curves are quadratic and have a more pronounced curvature than for $\beta=5 / 8$. We also observed that increasing the valence by knot insertion improves the curvature distribution for convex neighborhoods (see e.g. Figure 10). This is due to the increased symmetry of $v_{20}$ and the fact that, if a curve is $C^{1}$ at the central point and opposite curve segments are mirror images, then the curve is $C^{2}$. 


\begin{tabular}{|l|cccc|}
\hline$\beta$ & $3 / 8$ & $4 / 8$ & $5 / 8$ & $6 / 8$ \\
\hline$v_{20}$ & $(-1,5,29,68)^{\mathrm{t}}$ & $(-1,2,11,26)^{\mathrm{t}}$ & $(-1,1,5,12)^{\mathrm{t}}$ & $(-1,1 / 2,2,5)^{\mathrm{t}}$ \\
$\Delta^{2} v_{20}$ & $(18,15)^{\mathrm{t}}$ & $(6,6)^{\mathrm{t}}$ & $(2,3)^{\mathrm{t}}$ & $(0,3 / 2)^{\mathrm{t}}$ \\
\hline
\end{tabular}

Table I. Coefficients of $v_{20}$, the eigenvector of the zeroth Fourier mode to the eigenvalue $1 / 4$.

\section{CONCLUSION}

The algorithm just defined and analyzed is a polar cousin of Catmull-Clark subdivision. Its curvatures are bounded just as [Sabin 1991]. Its simplicity and the fact that the output consists of bicubic patches recommend bicubic polar subdivision as a useful addition to Catmull-Clark subdivision. This addition gives the designer more freedom just where Catmull-Clark subdivision encounters shape deficiencies, the valence is high or polar layout is natural. The paper [Myles et al. 2007] explains in detail how bicubic Catmull-Clark and bicubic polar subdivision can be combined for smooth object design such as in Figure 12.

\section{ACKNOWLEDGMENTS}

This work was supported in part by NSF Grants DMI-0400214 and CCF-0430891. Ashish Myles implemented the algorithm.

\section{REFERENCES}

Catmull, E. And Clark, J. 1978. Recursively generated B-spline surfaces on arbitrary topological meshes. Computer Aided Design 10, $350-355$.

DeRose, T., KASs, M., And Truong, T. 1998. Subdivision surfaces in character animation. In Siggraph 1998, Computer Graphics Proceedings, M. Cohen, Ed. ACM Press, 85-94.

Ginkel, I. And Umlauf, G. 2006. Loop subdivision with curvature control. In Proceedings of Symposium of Graphics Processing (SGP), June 26-28 2006, Cagliari, Italy, A. Scheffer and K. Polthier, Eds. ACM Press, 163-172.

KarČIaus Kas, K., Myles, A., And Peters, J. 2006. A $C^{2}$ polar jet subdivision. In Proceedings of Symposium of Graphics Processing (SGP), June 26-28 2006, Cagliari, Italy, A. Scheffer and K. Polthier, Eds. ACM Press, 173-180.

KARČIAUs KAs, K. AND PETERS, J. 2007. Surfaces with polar structure. Computing 79, 309-315.

Karciauskas, K., Peters, J., AND ReIF, U. 2004. Shape characterization of subdivision surfaces - case studies. Computer-Aided Geometric Design 21, 6 (july), 601-614.

Loop, C. 2002. Smooth ternary subdivision of triangle meshes. In Curve and Surface Fitting, Saint-Malo. Vol. 10(6). Nashboro Press, 3-6.

Morin, G., Warren, J., AND Weimer, H. 2001. A subdivision scheme for surfaces of revolution. Comp Aided Geom Design 18, 5, 483-502.

Myles, A., KarČIAuskas, K., And Peters, J. 2007. Extending Catmull-Clark subdivision and PCCM with Polar structures. In Proceedings of Pacific Graphics, Hawaii, M. Alexa, S. Gortler, and T. Ju, Eds. ACM Press, xx-xx.

PEters, J. AND REIF, U. 1998. Analysis of generalized B-spline subdivision algorithms. SIAM Journal on Numerical Analysis 35, 2 (Apr.), $728-748$

Peters, J. AND ShIUE, L.-J. 2004. Combining 4- and 3-direction subdivision. ACM Trans. Graph 23, 4, 980-1003.

REIF, U. 1995. A unified approach to subdivision algorithms near extraordinary vertices. Comp Aided Geom Design 12, $153-174$.

SAbIn, M. 1991. Cubic recursive division with bounded curvature. In Curves and Surfaces, L. S. P.J. Laurent, A. LeMéhauté, Ed. Academic Press, 411-414.

SCHAEFER, S. AND WARREN, J. D. 2005. On C ${ }^{2}$ triangle/quad subdivision. ACM Trans. Graph 24, 1, $28-36$.

StAm, J. AND Loop, C. T. 2003. Quad/triangle subdivision. Comput. Graph. Forum 22, 1, 79-86.

UMLAUF, G. 1999. Glatte freiformflächen und optimierte unterteilungsalgorithmen. Ph.D. thesis, Computer Science. 

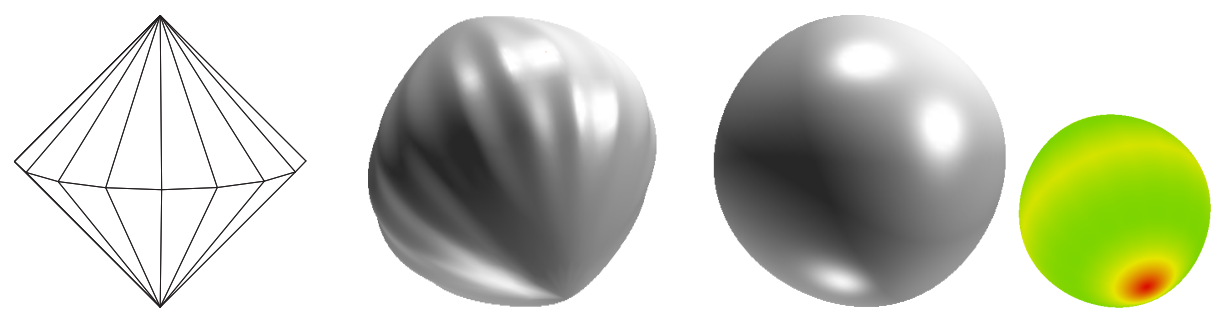

Fig. 7. Mirrored 16-sided pyramid. (left) control net; (middle and right) subdivision surfaces (three light sources) using (middle) Catmull-Clark subdivision, (right) polar bicubic subdivision (with Gauss-curvature shaded inset).
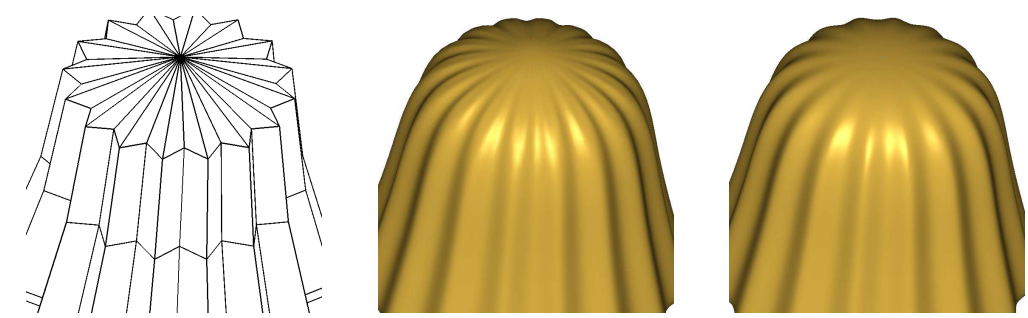

Fig. 8. Intended ripples: (left) Control net (middle) Catmull-Clark subdivision (note additional micro-ripples); (right) polar subdivision.
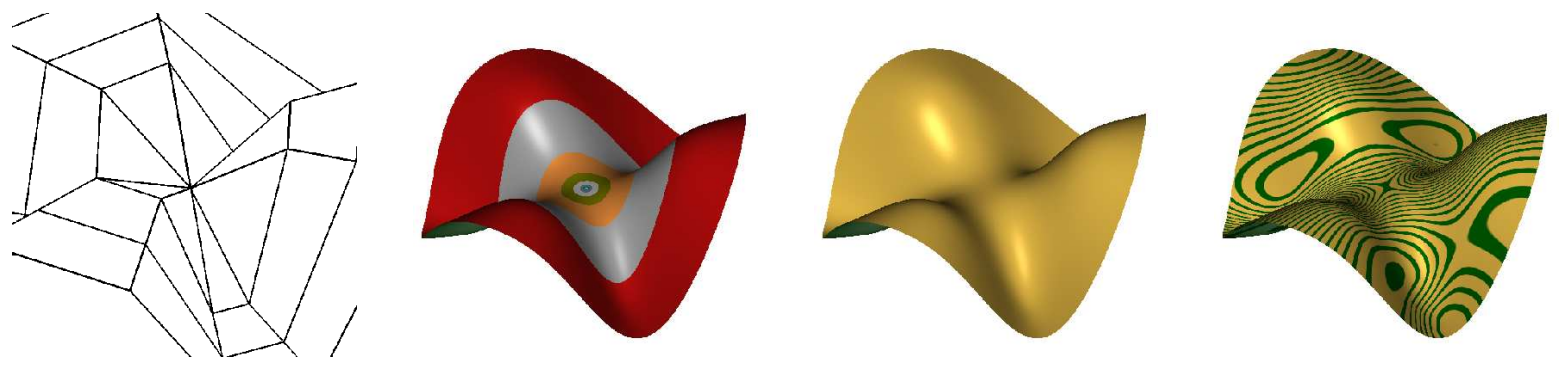

Fig. 9. Nonconvex polar net, nested surface rings of polar subdivision, shaded surface and reflection lines on the saddle.

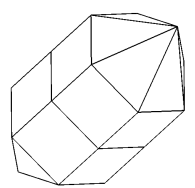

control net

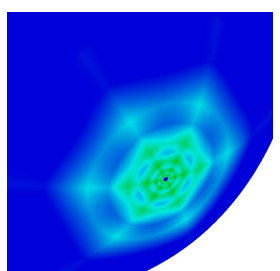

$n=6, \beta=\frac{5}{8}$

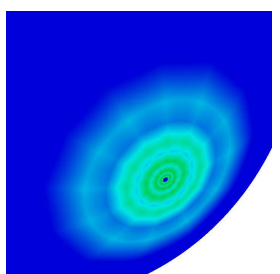

$n=12, \beta=\frac{5}{8}$

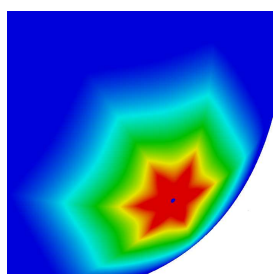

$n=6, \beta=\frac{1}{2}$

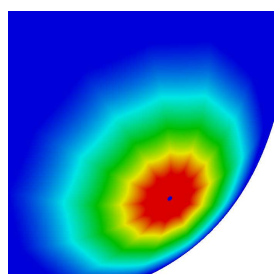

$n=12, \beta=\frac{1}{2}$

Fig. 10. The effect of changing the parameters $\beta$ and $n$ of bicubic polar subdivision on the everywhere positive Gauss-curvature of a capped cylinder. 

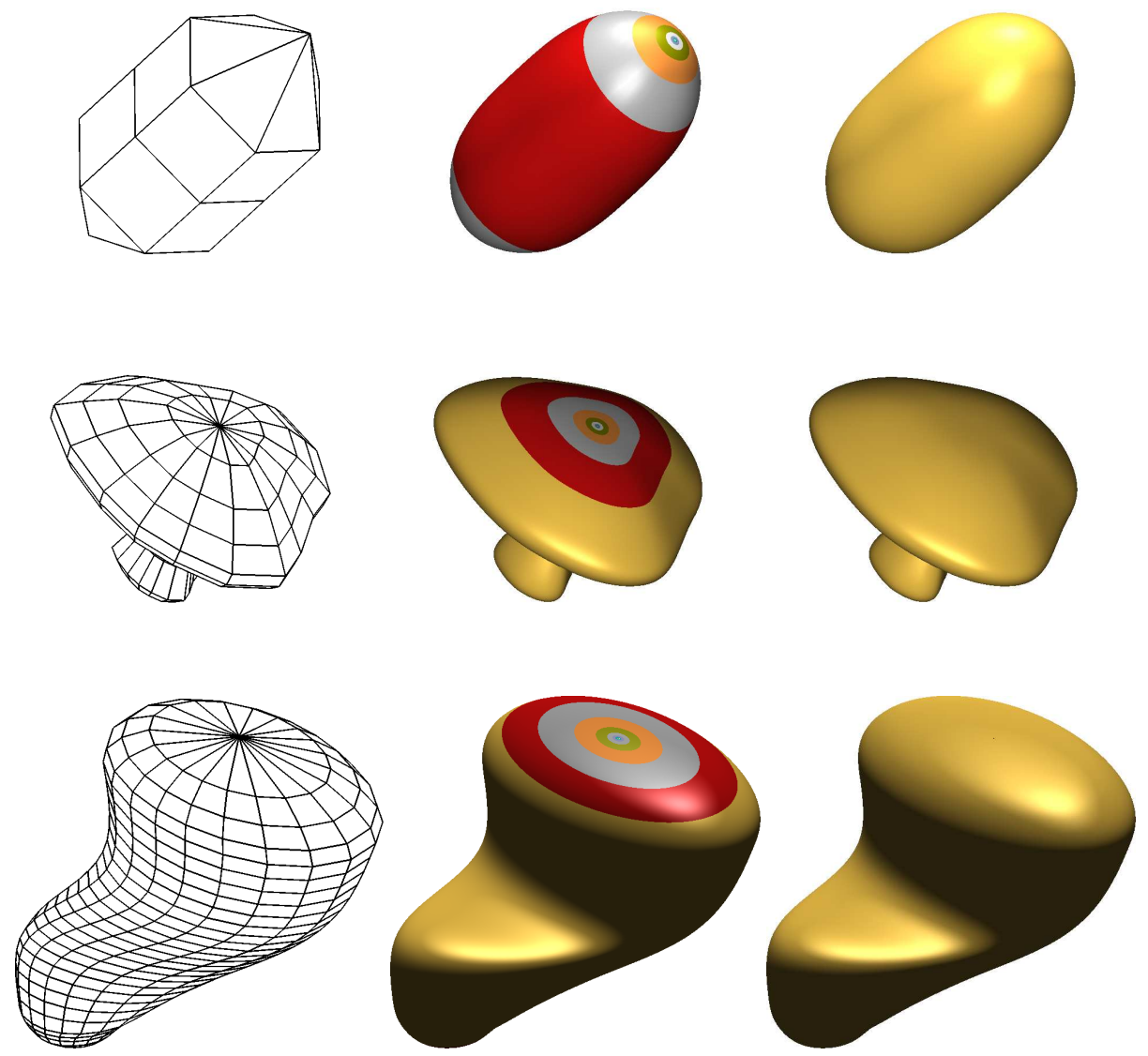

Fig. 11. (left) Sample meshes; (middle) bicubic surface rings; (right) Polar subdivision surfaces.
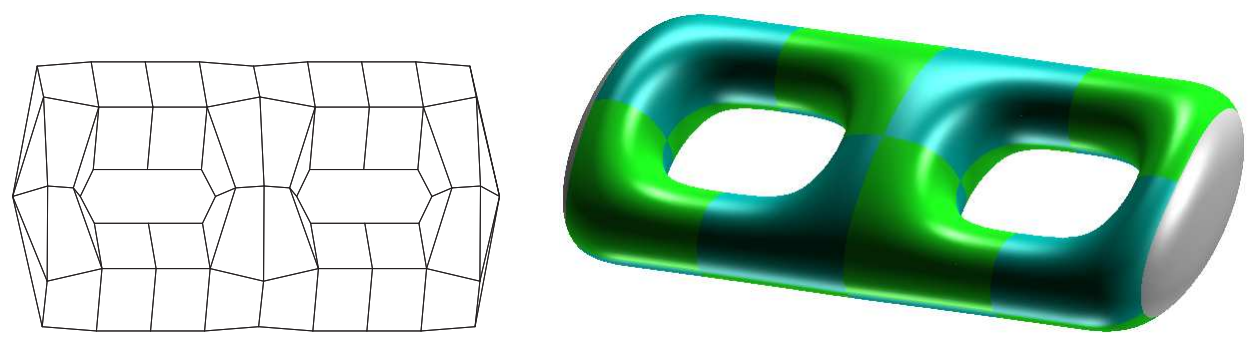

Fig. 12. Bicubic subdivision with Catmull-Clark rules applied where $n \neq 4$ quadrilaterals meet and polar rules where triangles meet (grey surfaces).

ACM Transactions on Graphics, Vol. V, No. N, Month 20YY. 